\title{
Predictive and Protective Factors for Partial Necrosis in DIEP Flap Breast Reconstruction
}

\author{
Does Nulliparity Bias Flap Viability?
}

\author{
Fabio Santanelli, MD, PhD, * Benedetto Longo, MD, PhD, * Barbara Cagli, MD, $\dagger$ \\ Pierfrancesco Pugliese, MD, * Michial Sorotos, MD, * and Guido Paolini, MD, PhD*
}

\begin{abstract}
Although success rate of deep inferior epigastric perforator (DIEP) flap breast reconstruction has greatly improved, complications still occasionally occur. Perfusion-related complications (PRCs) (ie, fat necrosis and partial flap necrosis) are the most frequent concern, affecting aesthetic final result of the reconstructed breast.

The aim of our study was to retrospectively investigate 287 consecutive DIEP flap breast reconstructions to investigate predictive and protective factors for PRCs.

From May 2004 to February 2012, 287 DIEP flap breast reconstructions were performed on 270 patients; 247 unilateral flaps, including Holm vascular zones I to III, were retrospectively selected and analyzed. Tobacco use, mean blood pressure over the first postoperative 48 hours, superficial epigastric vein drainage, medial/lateral row perforator, nulliparity, crystalloid versus combined crystalloid/colloid intravenous fluid infusion therapy, and learning curve were evaluated by univariate and multivariate logistic regression analyses.

Perfusion-related complications occurred 32 (12.9\%) times, 79 (31.9\%) patients were smokers, $48(19.4 \%)$ showed postoperative mean blood pressure less than $75 \mathrm{~mm} \mathrm{Hg}, 29$ (11.7\%) were nulliparous, and 173 (70\%) had superficial epigastric vein drainage. Selected perforators were $110(44.5 \%)$ from lateral row, 137 (55.5\%) from medial row; 91 (36.8\%) received crystalloid fluid infusion, whereas $156(63.2 \%)$ combined crystalloid/colloid fluid infusion. From univariate analysis emerged significance of nulliparity, perforator row and intravenous fluid infusion for PRC. Nevertheless, multivariate model confirmed only nulliparity as a significant risk factor $(P=0.029)$, although variable correlations to other predictors were found: both medial row perforator and combined crystalloid/colloid fluid infusion potentially decrease the PRC risk of $11.6 \%$ and $27.6 \%$, respectively. Learning curve did not show significant decrease of PRC risk over time.

Our study first proved nulliparity as a statistically significant predictor for PRCs in DIEP flap breast reconstruction, possibly due to different superficial abdominal perfusion between pluriparous and nulliparous women, with potential weaker pattern of perforators and smaller angiosomes in the latter. The choice of medial row perforators and combined crystalloid/colloid fluid infusion might reduce PRC risk.
\end{abstract}

Key Words: DIEP flap, partial necrosis, autologous breast reconstruction, perforator flap, nulliparity, risk factors

(Ann Plast Surg 2013;00: 00-00)

Received August 27, 2012, and accepted for publication, after revision, February $12,2013$.

From the *Plastic Surgery Unit, Sant'Andrea Hospital, and †Master's Student on Breast Reconstruction, School of Medicine and Psychology, "Sapienza" University of Rome, Rome, Italy.

Presented at the 23rd Annual EURAPS Meeting, May 24-26, 2012, Munich, Germany.

Conflicts of interest and sources of funding: none declared.

Reprints: Fabio Santanelli, MD, PhD, Azienda Ospedaliera Sant'Andrea, U.O.D Chirurgia Plastica, Via di Grottarossa 1035-1039, 00189 Rome, Italy. E-mail: fabio.santanelli@uniroma1.it.

Copyright (c) 2013 by Lippincott Williams \& Wilkins

ISSN: 0148-7043/13/0000-0000

DOI: 10.1097/SAP.0b013e31828d994d eep inferior epigastric perforator (DIEP) flap is one of the most popular choices for autologous breast reconstruction with lower abdominal tissue. The flap texture very similar to the normal breast, low donor-site morbidity, and postoperative pain are the main advantages of DIEP flap.

The most common concern of the DIEP flap is undoubtedly perfusion-related complications (PRCs) (ie, fat necrosis and partial flap necrosis) that can affect the aesthetic outcomes of the new breast while frequently leading to further surgical procedure to revise the reconstruction. The reported partial flap necrosis rates range from $6 \%$ to $17.4 \%{ }^{1}$ and although several attempts have been made to define predictors responsible for it, there is still no uniform consent. ${ }^{2-6}$

The aim of our study was to retrospectively investigate 287 consecutive DIEP flap breast reconstructions to investigate potential predictive and protective factors for PRCs.

\section{MATERIALS AND METHODS}

Between May 2004 and February 2012, 287 consecutive breast reconstructions with free DIEP flap were performed; all procedures were carried out by the senior author (F.S.). Bilateral reconstructions, using flaps with vascular zones I and II according to Holm et $\mathrm{al}^{7}$ and unilateral reconstruction using flaps with zones I, II, III, and IV were not included in the study, whereas only unilateral flaps with zones from I to III were included and retrospectively investigated.

Fat necrosis was detected at routine follow-up by ultrasound examination (minimum 6 months after surgery) by a specialized radiologist and considered positive in case of a solid, cystic lesion with or without well-defined margins, and minimum diameter of $1 \mathrm{~cm}^{3}$.

Partial flap necrosis was clinically defined as necrosis of the flap's skin, equal or larger than $1 \mathrm{~cm}^{2}$, and underlying fat by an independent plastic surgeon during the postoperative outpatient follow-up. Because both fat necrosis and partial flap necrosis were considered a result of poor flap perfusion, they were evaluated as cumulative total of complications.

A 2-team approach in 2 surgical steps was used in all patients, with simultaneous DIEP flap harvest from plastic surgeons and ablative procedure from oncologic surgeons at the first step, whereas flap insetting with microvascular anastomoses and simultaneous abdominal wall closure (by residents) at the second step. All flaps were harvested on either medial or lateral deep inferior epigastric artery branch through fascial incisions around each single perforator, splitting the rectus abdominis muscle, sparing the nerves if necessary. Surgeon selected best perforators by intraoperative direct visualization according to size and location, whereas a single perforator was used when venous perforator diameter was equal or larger than $1.5 \mathrm{~mm}$.

\section{Hypothetic Risk Factors}

The following predictors were considered as potential risk factors: tobacco use, mean blood pressure over the first postoperative 48 hours, super vein drainage through superficial inferior epigastric vein (SIEV), and the use of medial/lateral row perforator. The 
continuous variable mean blood pressure was dichotomized using its mean as cutoff point. Moreover, we included as potential predictor the intravenous crystalloid versus combined crystalloid/colloid fluid infusion during the first postoperative 24 hours. The combined crystalloid/colloid fluid administration consisted of intravenous infusion of a mean rate of $1.5 \mathrm{~mL} / \mathrm{kg}$ per hour started at the end of surgery, composed by the following items: (1) $500 \mathrm{~mL}$ of electrolytic solution $+5 \mathrm{~mL}$ of $33 \%$ glucose solution; (2) $500 \mathrm{~mL}$ of hydroxyethyl starch solution (Amidolite); (3) $500 \mathrm{~mL}$ of $0.9 \%$ isotonic sodium chloride solution $+5 \mathrm{~mL}$ of $33 \%$ glucose solution; (4) $500 \mathrm{~mL}$ of electrolytic solution $+5 \mathrm{~mL}$ of $33 \%$ glucose solution $+5 \mathrm{~mL}$ of ascorbic acid $(100 \mathrm{mg} / \mathrm{mL})$; and (5) $500 \mathrm{~mL}$ of $0.9 \%$ isotonic sodium chloride solution $+5 \mathrm{~mL}$ of $33 \%$ glucose solution.

Likewise, the repeated occurrence of zone III large necrosis in young nulliparous patients led us to hypothesize nulliparity as a potential risk factor for partial necrosis (Figs. 1 and 2).

Besides, because breast reconstruction with DIEP flap requires experience in microsurgery and perforator flaps harvest, we analyzed the equipe learning curve to highlight correlation between flap PRC and our surgical experience.

\section{Statistical Analysis}

Logistic regression analysis was used to identify risk factors related to PRC (ie, fat necrosis and partial flap necrosis). The impact of each factor was first determined using univariate logistic regression, then significant factors from univariate analysis were entered into multivariate logistic regression model with Firth's bias correction to adjust for the effects of all other variables. Multivariate analysis provides a natural and suitable framework for accounting of different effects on complications.

All analyses were performed using $\mathbf{R}$ ( $\mathrm{R}$ Development Core Team, 2009), and estimates of the models are obtained by the bayesglm function in the arm library. As coefficients in logistic regression can be challenging to interpret because of nonlinearity, we followed the "divide by 4 rule" 8 and interpreted the coefficients one at a time. Analysis of the learning curve by a multivariate logistic regression compared complications between first year group (2004-2005) and the other 7 chronological groups, 1 per year.

\section{RESULTS}

Among 287 DIEP flap breast reconstructions, 247 were identified as unilateral and including zone from I to III according to Holm (Table 1).

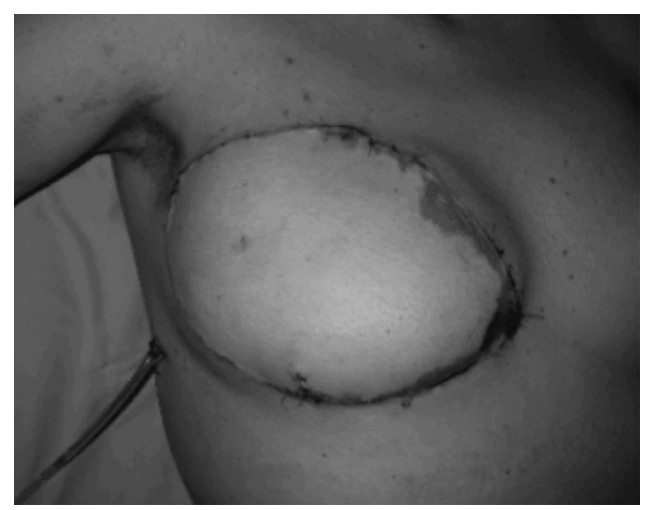

FIGURE 1. Partial necrosis to Holm zone III of a 35-year-old nulliparous patient who underwent right breast reconstruction with cutaneous-adipose $(12 \times 23 \mathrm{~cm})$ DIEP flap and anastomoses to circumflex scapular vessels and lateral thoracic vein.

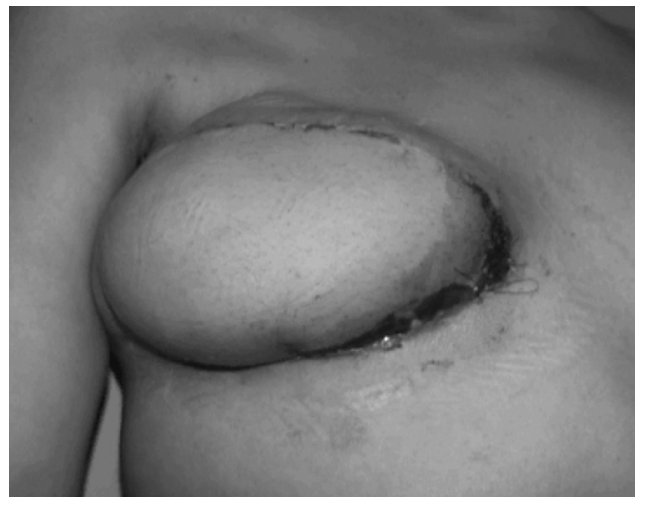

FIGURE 2. Partial necrosis to Holm zone III of a 39-year-old nulliparous patient who underwent right breast reconstruction with cutaneous-adipose $(14 \times 25 \mathrm{~cm})$ DIEP flap and anastomoses to circumf lex scapular vessels and subscapular vein.

Mean age at surgery was 51.3 years, ranging from 29 to 70 years. Postoperative follow-up ranged from 6 to 93 months with a mean value of 48 months.

Modified radical mastectomy was performed 165 (66.8\%) times, "skin sparing" mastectomy 46 (18.6\%), "nipple sparing" mastectomy on $23(9.3 \%)$, radical mastectomy on $5(2 \%)$, quadrantectomy once $(0.4 \%)$, and prosthesis replacement $7(2.8 \%)$ times.

Immediate breast reconstruction was performed on $201(81.4 \%)$ patients, delayed on $46(18.6 \%)$, the DIEP flaps were cutaneous-adipose $161(65.1 \%)$ times, adipose with a skin paddle on $59(23.8 \%)$, and purely adipose on $27(10.9 \%)$.

One perforator was used in $56(22.6 \%)$ flaps, $2(57.4 \%)$ in 142,3 $(18.6 \%)$ in 46 , and $4(1.2 \%)$ perforators in 3 flaps only (Table 2$)$.

Mean operative time was 5 hours and 23 minutes, including eventual contralateral symmetrization procedures.

A topographic summary of the deep inferior epigastric artery and vein and SIEV recipient sites is presented in Table 3.

\section{Flap PRCs}

Among 247 DIEP flaps, overall PRC occurred in 32 (12.9\%) cases, fat necrosis in $24(9.7 \%)$ flaps, and partial flap necrosis in 8 (3.2\%) cases. Among 32 PRCs, 29 (90.6\%) occurred in zone III, whereas $3(9.4 \%)$ in zone II (Table 4$)$. After demarcation, all patients were debrided under local or general anesthesia, and primary closure by DIEP flap advancement was accomplished.

\section{Partial Flap Necrosis and Risk Factors}

From univariate analysis, tobacco use, mean blood pressure over the first postoperative 48 hours, and super vein drainage through

\begin{tabular}{lc}
\hline TABLE 1. Patients and Procedure's Data & \\
\hline Mean age (range), y & $51.3(29-70)$ \\
Mean follow-up (range), mo & $48(6-93)$ \\
Mean operative time (range), h & $5.23(3.13-9.10)$ \\
Modified radical mastectomy & $165(66.8 \%)$ \\
Skin-sparing mastectomy & $46(18.6 \%)$ \\
Nipple-sparing mastectomy & $23(9.3 \%)$ \\
Radical mastectomy (Halsted/Patey) & $5(2 \%)$ \\
Prosthesis replacement & $7(2.8 \%)$ \\
Quadrantectomy & $1(0.4 \%)$ \\
Immediate reconstructions & $201(81.4 \%)$ \\
Delayed reconstructions & $46(18.6 \%)$ \\
\hline
\end{tabular}




\section{TABLE 2. DIEP Flaps' Data}

Overall number of DIEP flaps

Unilateral flaps including Holm zones I-II-III 287

Skin-adipose flaps

Adipose flaps with skin paddle

Purely adipose flaps

One perforator

Two perforators

Three perforators

Four perforators

SIEV were not statistically related to partial necrosis, whereas nulliparity, perforator row, and intravenous fluid infusion were all significantly related.

From multivariate model, although statistical significance was lost for all risk factors but nulliparity $(P=0.029), 2$ predictors showed a variable correlation to partial necrosis: both the use of medial row and combined crystalloid/colloid fluid infusion emerged as potential protective factor reducing the risk of partial necrosis of $11.6 \%$ and $27.6 \%$, respectively (Table 5 ).

Analysis of learning curve did not show any significant decrease of partial necrosis over time; nevertheless, equipe experience resulted as a protective factor reducing progressively partial necrosis risk of $2.5 \%$ to $23.9 \%$ from the second to the seventh year, respectively (Table 6).

\section{DISCUSSION}

During the last decade, DIEP flap has become one of the most preferred choices for autologous breast reconstruction because of excellent cosmetic results without consistent rectus muscle injury and reduced risk of abdominal bulge or hernia. The patient requires no intensive care unit after surgery nor long hospital stay, resulting in decreased social costs and high patient satisfaction.

The major concern of DIEP flap is undoubtedly PRC, occurring preferably in zones III and II according to Holm et al, ${ }^{7}$ and even if several attempts have been made to define risk factors for that, there is still no a uniform consent for them. Gill et $\mathrm{al}^{3}$ retrospectively examined 758 DIEP flaps and advocated smoking and adjuvant radiation therapy as independent predictors showing a significant association to fat necrosis; Scheer et $\mathrm{al}^{4}$ outlined that postoperative complications are related to patients comorbidities and personal risk factors; Hofer et $\mathrm{al}^{5}$ established no correlation to any risk factors but to a definite learning curve of the surgeon; Bozikov et $\mathrm{al}^{6}$ found patients with body mass index greater than $30 \mathrm{~kg} / \mathrm{m}^{2}$, flaps harvested on 1 perforator and microvascular revision surgery were significantly related to fat necrosis; and Peeters et $\mathrm{al}^{1}$ investigated other risk

\section{TABLE 3. Recipient Vessels}

\begin{tabular}{lcr}
\hline Recipient Vessels & Arteries & Veins \\
\hline Scapular Circumflex & 99 & 143 \\
Thoracodorsal & 74 & 120 \\
Internal mammary & 39 & 17 \\
Subscapular & 34 & 57 \\
Lateral thoracic & 1 & 102 \\
Cephalic & 0 & 1 \\
Axillary & 0 & 12 \\
Thoracodorsal serratus branch & 0 & 2 \\
Thoracoacromial & 0 & 8 \\
Total & 247 & 462 \\
\hline
\end{tabular}

\section{TABLE 4. DIEP Flap PRCs}

Overall number

$32(12.9 \%)$

Fat necrosis

$24(75 \%)$

Partial flap necrosis

$8(25 \%)$

Holm zone III necrosis

$29(90.6 \%)$

Holm zone II necrosis

$3(9.4 \%)$

factors and specifically radiation therapy but did not emerge any significant association to partial flap necrosis.

In our study, we assessed flap PRC rate and tried to highlight statistically related potential risk factors. In the literature, partial necrosis, investigated with ultrasound or clinical examination, is reported occurring in a range from $8.3 \%$ to $35 \%{ }^{1}$ and it compares with incidence of our case series $(12.9 \%)$.

From our statistical model, nulliparity $(P=0.029)$ emerged as a critical risk factor for partial necrosis, supporting our hypothesis, never investigated before, of a different superficial abdominal perfusion in nulliparous patients, being probably weaker the pattern of perforators and smaller the angiosome. Many studies on skin exposed to mechanical strain showed that such a stimulus invokes a cascade of events ultimately resulting in cellular growth and tissue regeneration through protein kinases, cytoskeletal structures, and growth factors. ${ }^{9-11}$ In particular, both arterial and venous patterns increase significantly in expanded skin, being collateral and communicating arteries and veins dilated and increased in number. During pregnancy, the growth of uterus, fetus, placenta, fetal membranes, and amniotic fluid causes a progressive stretching and expansion of the abdominal skin. Previously, autologous tissue expansion caused by pregnancy has been described for ventral hernia repair, for postburn reconstruction, and after tumor resection of the abdomen. ${ }^{12-14}$ The entity of such expansion can be appreciated considering that uterus weight is approximately $50 \mathrm{~g}$ when a woman is not pregnant and approximately $900 \mathrm{~g}$ by the end of pregnancy, whereas the fetus weight is approximately $3 \mathrm{~kg}$ and the placenta, fetal membranes, and amniotic fluid are approximately $1.8 \mathrm{~kg} .{ }^{15} \mathrm{As}$ a result, the overall mass of $5.7 \mathrm{~kg}$ could determine an abdominal skin expansion with consequent changing pattern of perforators and angiosomes becoming both larger than nulliparous women. It can be supposed that both the hormones stimulating tissue growth and the physiological mechanical expansion of pregnancy work like the delay phenomenon, making progressive dilation of choke vessels connecting 2 adjacent perforasomes, finally enlarging them to the size of "true" anastomoses. ${ }^{16,17}$ It follows that if pregnancy makes naturally "true" anastomoses, then the abdominal angiosome as well as the viable anatomical length of DIEP flap will be longer in women with previous pregnancies than nulliparous ones. On the basis of these findings, we now routinely discard zone IV in nulliparous patients while limiting as much as possible harvesting of zone III, possibly raised on medial row perforators. ${ }^{9}$

Tobacco role as a predictor of flap morbidity is controversial; indeed, some authors emphasized function of cigarettes' nicotine ${ }^{18,19}$ in activation of the sympathetic nervous system resulting in cutaneous vasoconstriction and leading to compromising of tissue capillary blood flow; others ${ }^{5,20}$ found that smoking, although it has adverse effects on random circulation flaps like mastectomy skin flaps, is not associated with a significant increase of free TRAM or DIEP flap complications. This compares with our data from which smoking did not emerge as a predictor $(P=0.0832)$ for partial necrosis in angiosomes-based perforator free flaps.

Hypotension on young fit people is generally considered to be a sign of good cardiovascular health not suggestive for compromised peripheral perfusion, as long as there are no preoperative symptoms (dizziness, blackouts, and so on); whereas during immediate 


\begin{tabular}{|c|c|c|c|c|c|}
\hline Risk Factors & No. Complications & $\%$ & $P^{*}$ & $P \dagger$ & Coefficients \\
\hline Nulliparous & $10 / 29$ & 34.5 & 0.0007 & 0.029 & 1.2358 \\
\hline Smokers & $15 / 79$ & 18.9 & 0.0832 & - & - \\
\hline Not smokers & $17 / 168$ & 10.1 & & & \\
\hline Lateral row & $20 / 110$ & 18.1 & 0.0454 & 0.3879 & -0.4635 \\
\hline Crystalloid infusion & $23 / 91$ & 25.2 & & & \\
\hline Mean blood pressure $<75 \mathrm{~mm} \mathrm{Hg}$ & $5 / 48$ & 10.4 & 0.7308 & - & - \\
\hline Mean blood pressure $\geq 75 \mathrm{~mm} \mathrm{Hg}$ & $27 / 199$ & 13.5 & & & \\
\hline SIEV & $20 / 173$ & 11.5 & 0.4288 & - & - \\
\hline No SIEV & $12 / 74$ & 16.2 & & & \\
\hline
\end{tabular}

*Univariate analysis.

$\dagger$ Multivariate analysis.

Bold text indicates significant $P$ values.

postoperative 48 hours, it could be advocated as potential risk factor for poor flap perfusion. However, the preservation of a good perfusion pressure ${ }^{21}$ rather than blood pressure is essential for flap survival, as there are other important determinants of peripheral perfusion. For this reason, during flap harvesting, reperfusion, and postoperative period, we tried to foresee mean pressure value greater than or equal to $100 \mathrm{~mm} \mathrm{Hg}$. Surprisingly, postoperative hypotension was not statistically significant $(P=0.7308)$ for partial necrosis.

Microcirculation of abdominal soft tissue has been elegantly studied by Moon and Taylor, ${ }^{22}$ and an insufficient venous outflow has been reported in several DIEP flaps ${ }^{23}$ because of insufficient interconnection between superficial and deep venous systems, without the intramuscular venous network compared to TRAM flap as described by Tran et $\mathrm{al}^{24}$ who suggest a second venous bypass. In our series, the use of a super vein drainage trough SIEV was not statistically related to partial necrosis $(P=0.4288)$.

The use of lateral or medial row perforators was found statistically associated to partial necrosis from univariate analysis $(P=0.0454)$ but not confirmed from multivariate model $(P=0.3879)$. Nevertheless, perforators from the lateral row seemed to be responsible factors potentially increasing the risk of flap PRC of $11.6 \%$. This result could be explained by the higher number of DIEP flaps $(90.6 \%)$ with partial necrosis in the distal portion of zone III farthest from the lateral perforators. This confirms the observations of Wong et $\mathrm{al}^{25}$ that lateral row perforators provide a worst perfusion to Holm zone III compared to medial row. On the basis of this result, we suggest to not select in advance medial row perforators to reduce flap morbidity unless a large area of Holm zone III is planned to be raised. Although some studies have shown that lateral perforators are dominant and easier to dissect due to a more perpendicular path through the rectus abdominis muscle, ${ }^{26,27}$ others consider their dissection exposing to higher risk of rectus abdominis motor nerve damage,${ }^{28,29}$ finally suggesting the use of medial row perforator vessels. ${ }^{30}$ From our experience, we believe that selection of perforators depends mutually on perforator location and size, and vascular territories included in the flap.

A key point in microsurgical free flap surgery is perioperative fluid management aiming to optimize flap perfusion. ${ }^{31}$ Although multivariate model confirmed it is not significant $(P=0.0697)$, from our series, the use of combined crystalloid/colloid fluid infusion emerged playing a potential protective role versus partial necrosis, reducing the risk of $27.6 \%$. It was shown that perfusion in a free flap tissue reduces up to $50 \%$ of the preoperative values during the first 6 to 12 hours after surgery. ${ }^{32}$ Moreover, being retained only $21 \%$ of crystalloids in the plasma volume and migrating the remaining $79 \%$ in the interstitial fluid volume after approximately 1 hour from their infusion, ${ }^{33,34}$ an excessive use could cause extreme flap edema and consequent further reduction of capillary blood flow, worsening the tissues ischemia. Rather, the use of hypervolemic hemodilution by combined crystalloid/colloid intravenous infusion increases cardiac output and, consequently, circulatory blood flow in the flap, being plasma levels of hydroxyethyl starch solution up to $80 \%$ at 6 hours after infusion. This pharmacokinetic profile allows for maintaining blood volume for at least 6 hours, obtaining a recommendable hematocrit value to approximately $30 \%$, over perfusing the flap in the most vulnerable phase, reducing tissue edema while assuring good pressure on the microcirculation and appropriate urine output.

Finally, contrary to other authors, ${ }^{5}$ our case series did not show significant association between learning curve and partial necrosis; nevertheless, the experience and confidence with the procedure, that is, perforator choice, flap drawing, dissection, etc, resulted as protective factors confirming a progression of learning from the second to the seventh year.

\section{LIMITATIONS}

Both the retrospective nature and observational character are the main limitations of our study. Reliable statistical results from multivariate logistic regression analyses in retrospective case series depend on minimum sample size and subject-to-variable ratio. Although several conventional rules of thumb currently exist, they are not valid and useful. ${ }^{35}$ However, the sample size (247 flaps) including only a specific type of flap (DIEP flaps including Holm zones I, II, and III) performed by a single surgeon are reliable indicators of a good statistical design. Nevertheless, we used Firth bias correction for

\begin{tabular}{lccc}
\hline TABLE 6. Learning Curve & & \\
\hline Patients & Coefficients (Risk Reduction) & $\boldsymbol{z}$ & $\boldsymbol{P}^{*}$ \\
\hline Group 1 (2005-2006) & $-0.1001(2.5 \%)$ & -0.154 & 0.87 \\
Group 2 (2006-2007) & $-0.6118(15.2 \%)$ & -0.967 & 0.33 \\
Group 3 (2007-2008) & $-0.4966(12.2 \%)$ & -0.754 & 0.45 \\
Group 4 (2008-2009) & $-0.9456(23.5 \%)$ & -1.395 & 0.16 \\
Group 5 (2009-2010) & $-0.9332(22.9 \%)$ & -1.299 & 0.18 \\
Group 6 (2010-2011) & $-0.8965(20.9 \%)$ & -1.1459 & 0.24 \\
Group 7 (2011-2012) & $-0.9630(23.9 \%)$ & -1.409 & 0.11 \\
\hline *Multivariate analysis. & & & \\
\hline
\end{tabular}


performing inference. Moreover, because the number of overall complications experienced in our series was relatively low ( $n=32$ events), we included only 6 predictors in multivariate model. As a result, predictors found related to PRCs could be confounded by other potential critical variables not measured by our model. Despite these limitations, this is the first study to highlight nulliparity as significant predictor for PRCs in DIEP flap breast reconstruction. Further anatomical studies investigating perforators and abdominal angiosomes of such patients will be essential to validate findings of our study. Lastly, although our approach to investigate the impact of combined crystalloid/colloid fluid infusion could be oversimplified by considering the mean infusion rate of $1.5 \mathrm{~mL} / \mathrm{kg}$ per hour, thus potentially resulting in loss of information regarding size and effect of the predictor, we found such intravenous fluid infusion replacement as an important protective factor for PRCs after DIEP flap breast reconstruction.

\section{CONCLUSIONS}

We first recognized nulliparity as a significant risk factor for PRCs in DIEP flap breast reconstruction, thus supporting our hypothesis of a different superficial abdominal perfusion between pluriparous and nulliparous patients, with potential weaker pattern of perforators and smaller angiosomes in the latter. Although such findings will need further anatomical studies to be confirmed, our results led us to consider nulliparous women as patients at higher risk for PRCs, hence deserving special care to flap design and harvesting so as not to include Holm zone IV, and limiting as much as possible Holm zone III. If zone III critical flap area is planned to be included in the flap, medial row perforators should be preferred to the lateral ones so as to reduce likelihood of postoperative flap PRCs.

Our study also confirmed different vascular territories between DIEP flaps based on medial row perforators and lateral ones, the latter providing worst perfusion to Holm zone III.

Finally, the need for hypervolemic hemodilution by combined crystalloid/colloid intravenous fluid infusion is considered worth to over perfuse the flap in its most vulnerable early phase. On the basis of our findings, we suggest intravenous administration of combined crystalloid/colloid fluid replacement with a mean rate of $1.5 \mathrm{~mL} / \mathrm{kg}$ per hour during the first postoperative 24 hours.

\section{ACKNOWLEDGMENTS}

The authors thank Maria Grazia Pittau, Associate Professor of Faculty of Statistics from "Sapienza" University of Rome, for the statistical analyses.

\section{REFERENCES}

1. Peeters WJ, Nanhekhan L, Van Ongeval C, et al. Fat necrosis in deep inferior epigastric perforator flaps: an ultrasound-based review of 202 cases. Plast Reconstr Surg. 2009;124:1754-1758.

2. Rogers NE, Allen RJ. Radiation effects on breast reconstruction with deep inferior epigastric perforator flap. Plast Reconstr Surg. 2002;109:1919-1924; discussion 1925-26.

3. Gill PS, Hunt JP, Guerra AB, et al. A 10-year retrospective review of 758 DIEP flaps for breast reconstruction. Plast Reconstr Surg. 2004;113: 1153-1160.

4. Scheer AS, Novak CB, Neligan PC, et al. Complications associated with breast reconstruction using a perforator flap compared with a free TRAM flap. Ann Plast Surg. 2006;56:355-358.

5. Hofer SOP, Damen THC, Mureau MAM, et al. A critical review of perioperative complications in 175 free deep inferior epigastric perforator flap breast reconstructions. Ann Plast Surg. 2007;59:137-142.

6. Bozikov K, Arnez T, Hertl K, et al. Fat necrosis in free DIEAP flaps: incidence, risk, and predictor factors. Ann Plast Surg. 2009;63:138-142.

7. Holm C, Mayr M, Höfter E, et al. Perfusion zones of the DIEP flap revisited: a clinical study. Plast Reconstr Surg. 2006;117:37-43.
8. Gelman A. Interpreting the logistic regression coefficient. In: Gelman S, Hill J, eds. Data Analysis Using Regression and Multilevel/Hierarchical Models Models. New York: Cambridge University Press; 2007; chapter 5:81-82.

9. De Filippo RE, Atale A. Stretch and growth: the molecular and physiologic influences of tissue expansion. Plast Reconstr Surg. 2002;109:2450-2462.

10. Mutaf M. Venous changes in expanded skin: a microangiographic and histological study in rabbits. Ann Plast Surg. 1996;37:75-83.

11. Lantieri LA, Martin-Garcia N, Wechsler J, et al. Vascular endothelial growth factor expression in expanded tissue: a possible mechanism of angiogenesis in tissue expansion. Plast Reconstr Surg. 1998;101:392-398.

12. Eric T, Walter L, Grant W. Pregnancy as a tissue expander in the repair of massive ventral hernia. Ann Plast Surg. 2000;45:431-433.

13. DiGregorio VR. Two unusual post-burn reconstructions using tissue expansion one exogenous and one endogenous. Ann Plast Surg. 1993;30:549-551.

14. Riordan C, Budny P, Regan P. Pregnancy as an autologous tissue expander for closure of an abdominal-wall defect. Br J Plast Surg. 2003;56:64-65.

15. Saladin KS. Anatomy and Physiology: The Unit Form and Function. 3rd ed. New York: McGraw-Hill Companies; 2004:1073; chapter 28.

16. Dhar S, Taylor GI. The delay phenomenon: the story unfolds. Plast Reconstr Surg. 1999;104:2079-2091.

17. Taylor GI, Corlett RJ, Dhar S, et al. The anatomical (angiosome) and clinical territories of cutaneous perforating arteries: development of the concept and designing safe flaps. Plast Reconstr Surg. 2011;127:1447-1459.

18. Benowitz NL. Clinical pharmacology of nicotine. Annu Rev Med. 1986;37:21.

19. Heliovaara M, Karvonen MJ, Punsar S. Smoking, carbon monoxide and atherosclerotic disease. Br Med J. 1978;1:268.

20. Chang DW, Reece GP, Wang B, et al. Effect of smoking on complications in patients undergoing free TRAM flap breast reconstruction. Plast Reconstr Surg. 2000;105:2374-2380.

21. Al-Benna S, Grob M. Primary hypotension: a contraindication to free tissue transfer? Plast Reconstr Surg. 2006;117:2509.

22. Moon HK, Taylor GI. The vascular anatomy of rectus abdominis musculocutaneous flaps based on the deep inferior epigastric system. Plast Reconstr Surg. 1988;82:815-832.

23. Wechselberger G, Schoeller T, Bauer T, et al. Venous superdrainage in deep inferior epigastric perforator flap breast reconstruction. Plast Reconstr Surg. 2001;108:162.

24. Tran NV, Buchel EW, Convery PA. Microvascular complications of DIEP flaps. Plast Reconstr Surg. 2007;119:1307-1405.

25. Wong C, Saint-Cyr M, Mojallal A, et al. Perforasomes of the DIEP flap: vascular anatomy of the lateral versus medial row perforators and clinical implications. Plast Reconstr Surg. 2010;125:772-782.

26. Blondeel PN, Arnstein M, Verstraete K, et al. Venous congestion and blood flow in free transverse rectus abdominis myocutaneous and deep inferior epigastric perforator flaps. Plast Reconstr Surg. 2000;106:1295.

27. Vandevoort M, Vranckx JJ, Fabre G. Perforator topography of the deep inferior epigastric perforator flap in 100 cases of breast reconstruction. Plast Reconstr Surg. 2002;109:1912-1918.

28. Rozen WM, Ashton MW, Murray AC, et al. Avoiding denervation of rectus abdominis in DIEP flap harvest: the importance of medial row perforators. Plast Reconstr Surg. 2008;122:710-716.

29. Rozen WM, Ashton MW, Murray AC, et al. Avoiding denervation of rectus abdominis in DIEP flap harvest II: an intraoperative assessment of the nerves to rectus. Plast Reconstr Surg. 2008;122:1321-1325.

30. Rozen WM, Ashton MW, Le Roux CM, et al. The perforator angiosome: a new concept in the design of deep inferior epigastric artery perforator flaps for breast reconstruction. Microsurgery. 2010;30:1-7.

31. Zhong $\mathrm{T}$, Neinstein $\mathrm{R}$, Massey $\mathrm{C}$, et al. Intravenous fluid infusion rate in microsurgical breast reconstruction: important lessons learned from 354 free flaps. Plast Reconstr Surg. 2011;128:1153-1160.

32. Hagau N, Longrois D. Anesthesia for free vascularised tissue transfer. Microsurgery. 2009;29:161-167.

33. Ernest D, Belzberg AS, Dodek PM. Distribution of normal saline and $5 \%$ albumin infusions in septic patients. Crit Care Med. 1999;27:46-50.

34. Weil MH. Crystalloids, colloids and fluid compartments. Crit Care Med. 1999;27:3.

35. MacCallum RC, Widaman KF, Zhang S, et al. Sample size in factor analysis. Psychol Methods. 1999;4:84-89. 